\title{
GLOBAL EXISTENCE AND BLOW-UP FOR SEMILINEAR DAMPED WAVE EQUATIONS IN THREE SPACE DIMENSIONS
}

\author{
MASAKAZU KATO \\ Muroran Institute of Technology \\ 27-1 Mizumoto-cho, Muroran 050-8585, Japan \\ MiKu SAKURABA \\ Sapporo Hokuto High School \\ 1-10 Kita 15, Higashi 2, Higashi-ku, Sapporo 065-0015, Japan
}

॥

\begin{abstract}
We consider initial value problem for semilinear damped wave equations in three space dimensions. We show the small data global existence for the problem without the spherically symmetric assumption and obtain the sharp lifespan of the solutions. This paper is devoted to a proof of the Takamura's conjecture in [5] on the lifespan of solutions.
\end{abstract}

Keywords: Semilinear damped wave equation, Blow-up, Lifespan, three space dimensions.

\section{Introduction}

In this paper, we consider the Cauchy problem for semilinear damped wave equations

$$
\begin{aligned}
& v_{t t}(x, t)-\Delta v(x, t)+\frac{\mu}{(1+t)^{\beta}} v_{t}(x, t)=|v(x, t)|^{p} \quad \text { for } \quad(x, t) \in \mathbb{R}^{n} \times[0, \infty), \\
& v(x, 0)=\epsilon f(x), \quad v_{t}(x, 0)=\epsilon g(x) \quad \text { for } \quad x \in \mathbb{R}^{n}
\end{aligned}
$$

where $n \in \mathbb{N}, p>1, \beta \in \mathbb{R}, \mu>0$ and $\epsilon>0$. Let $\rho \geq 1$ and we assume that

$$
\operatorname{supp}\{f(x), g(x)\} \subset\left\{x \in \mathbb{R}^{n}|| x \mid \leq \rho\right\} .
$$

AMS Subject Classifications: 35L71, 35E15, 35A01. 
When $\beta=0$, (1.1) is a important mathematical model to describe the wave propagation with friction and the heat conduction with finite speed of propagation. For example, magnetohydrodynamics, viscoelasticity, and flood flow with friction are expressed by the damped wave equations. When $\beta \neq 0$, the equation is a model to describe the microwave drying processes for hygroscopic materials and the vibrations of a strings with variable tension and density, such as the time-dependent telegraph equation (see [7]).

It is interesting to find a critical exponent $p_{c}(n)$ such that if $p>p_{c}(n)$, then the small data global existence holds for (1.1) and (1.2), while if $1<p \leq p_{c}(n)$, then small data blowup occurs. Moreover, for the blowup case, our purpose is to derive estimates of upper and lower bounds of the lifespan which is the maximal existence time of the solution. In this paper, we study small data global existence and blowup for (1.1) and (1.2) especially with $n=3, \beta=1$ and $\mu=2$. Before we proceed to our problem, we recall some known results.

For the case $\beta \in(-1,1)$, the global existence has been obtained by D'Abbicco, Lucente and Ressig [4] showed the global existence for

$$
p_{F}(n)<p< \begin{cases}\infty & \text { for } n=1,2 \\ n /(n-2) & \text { for } n \geq 3\end{cases}
$$

where $p_{F}(n)=1+2 / n$ is the critical power for semilinear heat equation, $u_{t}-\Delta u=|u|^{p}$. For $\beta \in(-1,1)$ and $1<p \leq p_{F}(n)$, the lifespan estimates were obtained by Ikeda and Ogawa [10], Fuhiwara, Ikeda and Wakasugi [8], Ikeda and Inui [9]. Then, it is known that the critical exponent is $p_{F}(n)$ for $\beta \in(-1,1)$.

When $\beta=1$, the coefficient $\mu$ plays a crucial role in this case. Indeed, the critical exponent depends on $\mu$. D'Abbicco and Lucente [2], and D'Abbicco [1] have showed that the critical power is $p_{F}(n)$ when

$$
\mu \geq \begin{cases}5 / 3 & \text { for } n=1 \\ 3 & \text { for } n=2 \\ n+2 & \text { for } n \geq 3\end{cases}
$$

while Wakasugi [17] has obtained blow up for $1<p \leq p_{F}(n)$ and $\mu \geq 1$, or $1<p \leq$ $p_{F}(n+\mu-1)$ and $0<\mu<1$. We see that if $0<\mu<1$, then $p_{c}(n) \geq p_{F}(n+\mu-1)>p_{F}(n)$.

When $\beta=1$ and $\mu=2$, by setting $u(x, t)=(1+t) v(x, t)$, we can rewrite (1.1) and (1.2) as the following semilinear wave equations

$$
\begin{aligned}
& \square u(x, t)=(1+t)^{-(p-1)}|u(x, t)|^{p} \quad \text { for } \quad(x, t) \in \mathbb{R}^{n} \times[0, \infty), \\
& u(x, 0)=\epsilon f(x), \quad u_{t}(x, 0)=\epsilon\{f(x)+g(x)\} \quad \text { for } \quad x \in \mathbb{R}^{n} .
\end{aligned}
$$


Due to this observation, D'Abbicco, Lucente and Ressig [5] have determined a critical power

$$
p_{c}(n)=\max \left\{p_{F}(n), p_{S}(n+2)\right\} \quad \text { for } n \leq 3,
$$

where $p_{S}(n)$ is called Strauss exponent which is the critical exponent of semilinear wave equations $w_{t t}-\Delta w=|w|^{p}$. We remark that

$$
p_{S}(n)=\frac{n+1+\sqrt{n^{2}+10 n-7}}{2(n-1)} \quad(n \geq 2),
$$

and $p_{S}(n)$ be the positive root of the quadratic equation

$$
\gamma(p, n):=2+(n+1) p-(n-1) p^{2}=0 .
$$

D'Abbicco and Lucente [3] have also showed the global existence for $p_{S}(n+2)<p<$ $1+2 / \max \{2,(n-3) / 2\}$ to odd and higher dimensions $(n \geq 5)$ under the spherically symmetric assumption. For $n=3, p_{c}(3)=p_{S}(5)$ follows from (1.6). In the blowup case $1<p \leq p_{F}(n)$, Wakasugi [17, 18] has showed that the upper bound of life span of the solutions for (1.4) and (1.5) is

$$
T(\epsilon) \leq C \epsilon^{-(p-1) /\{2-n(p-1)\}} .
$$

In [5], for $n=3$ and $(f, g) \neq(0,0)$, they remark the following Takamura's conjecture.

$$
T(\epsilon) \sim \begin{cases}C \epsilon^{-\frac{2 p(p-1)}{\gamma(p, 5)}} & \left(1<p<p_{S}(5)\right) \\ \exp \left(C \epsilon^{-p(p-1)}\right) & \left(p=p_{S}(5)\right)\end{cases}
$$

Our main goal in this paper is to obtain the lifespan estimate (1.8) for (1.4) and (1.5) with $n=3$. Also, our purpose is to show the small data global existence for $p>p_{S}(5)$ without the symmetric condition.

We put

$$
m(p)=\left\{\begin{array}{ll}
1 & (1<p<2) \\
2 & (p \geq 2)
\end{array} .\right.
$$

We think of $C^{m(p)}$-solutions of the following integral equation associated with (1.4) and (1.5):

$$
u(x, t)=u^{0}(x, t)+L\left[|u|^{p}\right](x, t), \quad(x, t) \in \mathbb{R}^{3} \times[0, \infty),
$$

where

$$
L[w](x, t)=\frac{1}{4 \pi} \int_{0}^{t}(t-s) d s \int_{|\eta|=1}(1+s)^{-(p-1)} w(x+(t-s) \eta, s) d \omega_{\eta}
$$


for $w \in C\left(\mathbb{R}^{3} \times[0, \infty)\right)$ and $u^{0}$ is a solution to the linear wave equations

$$
\begin{aligned}
& u_{t t}(x, t)-\Delta u(x, t)=0, \quad(x, t) \in \mathbb{R}^{3} \times[0, \infty), \\
& u(x, 0)=\epsilon f(x), \quad u_{t}(x, 0)=\epsilon\{f(x)+g(x)\}, \quad x \in \mathbb{R}^{3} .
\end{aligned}
$$

We remark that if $u \in C^{2}\left(\mathbb{R}^{3} \times[0, \infty)\right)$ is the solution of (1.10) with $p \geq 2$, then $u$ is the classical solution to the initial value problem (1.4) and (1.5) (See Lemma I in [13].).

To state our results, we define the lifespan $T(\epsilon)$ of the solution of (1.10) by

$$
T(\epsilon):=\sup \left\{T \in[0, \infty) \mid \text { There exists a unique solution } u \in C^{1}\left(\mathbb{R}^{3} \times[0, T)\right) \text { of }(\underline{1.10}) .\right\}
$$

for arbitrarily fixed $(f, g)$.

In the following theorem, we establish the global existence without the spherically symmetric assumption.

Theorem 1.1. Let $n=3, p>p_{S}(5)=\frac{3+\sqrt{17}}{4}, f \in C_{0}^{2+m(p)}\left(\mathbb{R}^{3}\right)$ and $g \in C_{0}^{1+m(p)}\left(\mathbb{R}^{3}\right)$, where $m(p)$ is given by (1.9). If $\epsilon$ is sufficiently small, then (1.10) has a unique global solution $u \in C^{m(p)}\left(\mathbb{R}^{3} \times[0, \infty)\right)$.

REMARK 1.2. In [5], for $p>p_{S}(5)$, they have showed the global existence in $C\left(\mathbb{R}^{3} \times\right.$ $[0, \infty)) \cap C^{2}\left(\mathbb{R}^{3} \backslash\{0\} \times[0, \infty)\right)$ with the radial symmetric condition.

We obtain the lower bound of the lifespan as follows.

Theorem 1.3. Let $n=3,1<p \leq p_{S}(5), f \in C_{0}^{3}\left(\mathbb{R}^{3}\right)$ and $g \in C_{0}^{2}\left(\mathbb{R}^{3}\right)$. There exist positive constants $A$ and $\epsilon_{0}$ such that the solution $u \in C^{1}\left(\mathbb{R}^{3} \times[0, \infty)\right)$ of (1.10) exists as far as

$$
T \leq \begin{cases}A \epsilon^{-\frac{2 p(p-1)}{\gamma(p, 5)}} & \left(1<p<p_{S}(5)\right) \\ \exp \left(A \epsilon^{-p(p-1)}\right) & \left(p=p_{S}(5)\right)\end{cases}
$$

for $0<\epsilon \leq \epsilon_{0}$.

The following theorem shows the optimality of the lower bound of Theorem 1.3. Hence, we solve the Takamura's conjecture.

Theorem 1.4. Let $n=3,1<p \leq p_{S}(5), f \in C_{0}^{3}\left(\mathbb{R}^{3}\right), g \in C_{0}^{2}\left(\mathbb{R}^{3}\right)$. We assume that $f \equiv 0$ and $g \geq 0(g \not \equiv 0)$. There exists a positive constant $\epsilon_{0}$ such that for $0<\epsilon \leq \epsilon_{0}$, the solution of (1.10) blows up in a finite time $T(\epsilon)$. Moreover, there exists a positive constant $B$ independent of $\epsilon$ such that

$$
T(\epsilon) \leq\left\{\begin{array}{ll}
B \epsilon^{-\frac{2 p(p-1)}{\gamma(p, 5)}} & \left(1<p<p_{S}(5)\right) \\
\exp \left(B \epsilon^{-p(p-1)}\right) & \left(p=p_{S}(5)\right)
\end{array} .\right.
$$


REMARK 1.5. Recently, Ikeda and Sobajima [11] obtain improvements on the lifespan estimates for (1.4) and (1.5) with $n \geq 1$. In particular, when $n=3$, by using the test function method, they have showed the estimates as follows.

$$
T(\epsilon) \leq \begin{cases}C \epsilon^{-1-\delta} & \left(p_{F}(3)<p<p_{S}(7)\right) \\ C \epsilon^{-\frac{2 p(p-1)}{\gamma(p, 5)}-\delta} & \left(p_{S}(7) \leq p<p_{S}(5)\right) \\ \exp \left(C \epsilon^{-p(p-1)}\right) & \left(p=p_{S}(5)\right)\end{cases}
$$

with arbitrary small $\delta>0$.

REMARK 1.6. Wakasa [16] had obtained the optimal life span estimate for (1.10) with $n=1$. The study of the lifespan of solutions to the semilinear damped wave equation with the general variable coefficient has been studied by many mathematicians (see [14, 15] and refer to the references cited therein).

The article is organized as follows. In Section 2, we show the decay estimate for the solution of linear wave equations and give the estimate of the integral operator (1.11). Then, we prove Theorem 1.1 and Theorem 1.3. The global existence and lower bounds of the life span will be obtained by the weighted $L^{\infty}-L^{\infty}$ estimates introduced in Lemma 2.2 and Lemma 2.3. In Section 3, we prove Theorem 1.4. We will show the upper bounds of lifespan by using the iteration argument and the slicing method.

\section{Global existence and Lower bound of the lifespan}

In this section, we prove Theorem 1.1 and Theorem 1.3. We shall construct a solution of integral equation (1.10), employing the iteration method in [13] and [12].

First, we study the decay estimate of solution for the homogeneous wave equation (1.12) and (1.13). The solution can be expressed by

$$
u^{0}(x, t)=\epsilon \frac{t}{4 \pi} \int_{|\xi|=1}\{f(x+t \xi)+g(x+t \xi)\} d \omega_{\xi}+\epsilon \frac{\partial}{\partial t}\left\{\frac{t}{4 \pi} \int_{|\xi|=1} f(x+t \xi) d \omega_{\xi}\right\} .
$$

We prepare the following decay estimate of solution of free wave equations.

Lemma 2.1. Assume a support property (1.3). Then, there exists a constant $\gamma$ such that for $\nu=0,1,2,3$ we have

$$
\rho^{|\alpha|}\left|D_{x}^{\alpha} u^{0}(x, t)\right| \leq \epsilon \gamma\left(\frac{t+\rho}{\rho}\right)^{-1} N_{\nu} \quad \text { for } \quad x \in \mathbb{R}^{3}, t \geq 0,|\alpha| \leq \nu
$$

where

$$
N_{\nu}=\sum_{|\alpha| \leq \nu+2} \sup _{x \in \mathbb{R}^{3}} \rho^{|\alpha|}\left|D_{x}^{\alpha} f(x)\right|+\sum_{|\beta| \leq \nu+1} \sup _{x \in \mathbb{R}^{3}} \rho^{|\beta|+1}\left\{\left|D_{x}^{\beta} f(x)\right|+\left|D_{x}^{\beta} g(x)\right|\right\} .
$$


Moreover, it holds

$$
u^{0}(x, t)=0 \quad \text { for }|t-| x|| \geq \rho .
$$

Proof. This well-known fact can be found in (45c) and (45d) of [13]. We shall omit the proof.

Next, we estimate the integral operator (1.11). We denote a weighted $L^{\infty}$ norm by

$$
\|u\|=\sup _{\substack{x \in \mathbb{R}^{3} \\ 0 \leq t<T}} w(|x|, t)|u(x, t)|
$$

Here,

$$
w(r, t)=\left\{\begin{array}{ll}
\rho^{-2(p-1)}(t+r+2 \rho)^{q}(t-r+2 \rho)^{\bar{q}} & \left(p \neq \frac{3}{2}\right) \\
\rho^{-1}(t+r+2 \rho)\left\{\log \frac{2(t+r+2 \rho)}{t-r+2 \rho}\right\}^{-1} & \left(p=\frac{3}{2}\right)
\end{array},\right.
$$

where

$$
q=\max \{2(p-1), 1\} \quad \text { and } \quad \bar{q}=\max \{0,2 p-3\} .
$$

We define $\bar{v}(r, t)=\sup _{\substack{x \\|x|=r}}|v(x, t)|$. From (2.3) and (2.4), it follows that

$$
\bar{u}(\lambda, s) \leq\|u\| \times\left\{\begin{array}{ll}
\left(\frac{s+\lambda+2 \rho}{\rho}\right)^{-q}\left(\frac{s-\lambda+2 \rho}{\rho}\right)^{-\bar{q}} & \left(p \neq \frac{3}{2}\right) \\
\left(\frac{s+\lambda+2 \rho}{\rho}\right)^{-1} \log \frac{2(s+\lambda+2 \rho)}{s-\lambda+2 \rho} & \left(p=\frac{3}{2}\right)
\end{array} .\right.
$$

The following a priori estimate plays a key role in the iteration method.

Lemma 2.2. Let $p>1$. Assume that $u \in C\left(\mathbb{R}^{3} \times[0, T)\right)$ with $\operatorname{supp} u \subset\{(x, t) \in$ $\left.\mathbb{R}^{3} \times[0, T)|| x \mid \leq t+\rho\right\}$. Then, it holds

$$
\left\|L\left[|u|^{p}\right]\right\| \leq C_{1} \rho^{2}\|u\|^{p} D(T),
$$

where $D(T)$ is defined by

$$
D(T)= \begin{cases}\left(\frac{2 T+3 \rho}{\rho}\right)^{\gamma(p, 5) / 2} & \left(1<p<p_{S}(5)\right) \\ \log \left(\frac{T+2 \rho}{\rho}\right) & \left(p=p_{S}(5)\right) \\ 1 & \left(p>p_{S}(5)\right)\end{cases}
$$

Proof. From the assumption in Lemma 2.2, we obtain

$$
\bar{u}(\lambda, s)=0 \quad \text { for } \lambda \geq s+\rho .
$$


Since $\rho \geq 1$, we have from Lemma II in [13] and (2.9)

$$
\begin{aligned}
\left|L\left[|u|^{p}\right](x, t)\right| & \leq \int_{0}^{t} d s \int_{I} \frac{\lambda}{2 r}\left(\frac{s+\rho}{\rho}\right)^{-(p-1)} \bar{u}(\lambda, s)^{p} d \lambda \\
& =\iint_{\tilde{R}(r, t)} \frac{\lambda}{2 r}\left(\frac{s+\rho}{\rho}\right)^{-(p-1)} \bar{u}(\lambda, s)^{p} d \lambda d s
\end{aligned}
$$

where $r=|x|, I=[|r-t+s|, r+t-s] \cap[|r-t+s|, s+\rho]$ and

$$
\tilde{R}(r, t)=\{(\lambda, s) \mid 0 \leq s \leq t, \lambda \in I\} .
$$

It is clear that (2.7) follows from the basic estimate:

$$
\left|L\left[|u|^{p}\right](x, t)\right| \leq C \rho^{2}\|u\|^{p} w(r, t)^{-1} D(T) .
$$

We show (2.11) in the following two sets separately. Put

$$
S_{1}=\{(r, t) \mid t-\rho \leq r \leq t+\rho\}, \quad S_{2}=\{(r, t) \mid 0 \leq r \leq t-\rho\} .
$$

First, we consider the case $(r, t) \in S_{1}$. We see

$$
1 \leq \frac{t-r+2 \rho}{\rho} \leq 3 \quad \text { and } \quad \frac{t+r+2 \rho}{3} \leq t+\rho \quad \text { for }(r, t) \in S_{1} .
$$

From (2.6) and (2.13), we get

$$
\bar{u}(\lambda, s) \leq\|u\|\left(\frac{s+\rho}{\rho}\right)^{-q} \eta(t) \quad \text { for }(\lambda, s) \in S_{1}
$$

where

$$
\eta(t)=\left\{\begin{array}{ll}
1 & \left(p \neq \frac{3}{2}\right) \\
\log \frac{6(t+\rho)}{\rho} & \left(p=\frac{3}{2}\right)
\end{array} .\right.
$$

From p.255 in [13], for $0 \leq s \leq t$ and $(r, t) \in S_{1}$, we find

$$
\frac{1}{2 r} \int_{I} \lambda d \lambda \leq \frac{12 \rho(s+\rho)}{t+\rho}
$$

Noticing that $\tilde{R}(r, t) \subset S_{1}$ and substituting (2.14) into (2.10), we obtain from (2.16),$(2.5)$ 
and (2.13)

$$
\begin{aligned}
L\left[|u|^{p}\right](x, t) & \leq\|u\|^{p} \eta(t)^{p} \int_{0}^{t} d s\left(\frac{s+\rho}{\rho}\right)^{-(q+1) p+1} \int_{I} \frac{\lambda}{2 r} d \lambda \\
& \leq \frac{12 \rho^{2}\|u\|^{p} \eta(t)^{p}}{t+\rho} \int_{0}^{t}\left(\frac{s+\rho}{\rho}\right)^{-(q+1) p+2} d s \\
& \leq C \rho^{2}\|u\|^{p} \eta(t)^{p+1}\left(\frac{t+\rho}{\rho}\right)^{-1+\max \left\{-2 p^{2}+p+3,0\right\}} \\
& \leq C \rho^{2}\|u\|^{p}\left(\frac{t+r+2 \rho}{\rho}\right)^{-q} \times\left\{\begin{array}{ll}
\left(\frac{T+\rho}{\rho}\right)^{-2 p^{2}+3 p} & \left(1<p<\frac{3}{2}\right) \\
\left\{\log \frac{6(T+\rho)}{\rho}\right\}^{\frac{5}{2}} & \left(p=\frac{3}{2}\right) \\
1 & \left(p>\frac{3}{2}\right)
\end{array} .\right.
\end{aligned}
$$

Hence, we get from (2.13) and (2.8)

$$
L\left[|u|^{p}\right](x, t) \leq C \rho^{2}\|u\|^{p}\left(\frac{t+r+2 \rho}{\rho}\right)^{-q}\left(\frac{t-r+2 \rho}{\rho}\right)^{-\bar{q}} D(T) \quad \text { in } S_{1} .
$$

Therefore we get (2.11) for $(r, t) \in S_{1}$.

Next, we consider the case $(r, t) \in S_{2}$. Introducing new variables of integration

$$
\alpha=s+\lambda, \quad \beta=s-\lambda
$$

For $\alpha+\beta \geq 0$ and $\beta \geq-\rho$, we have

$$
\begin{aligned}
\frac{s+\rho}{\rho} & \geq \frac{\alpha+\beta+4 \rho}{4 \rho} \\
& \geq \frac{\alpha+2 \rho}{4 \rho} .
\end{aligned}
$$

Since $\left|\frac{\partial(\lambda, s)}{\partial(\alpha, \beta)}\right|=1 / 2$, it follows from (2.10) and (2.19) that

$$
\left|L\left[|u|^{p}\right](x, t)\right| \leq \int_{-\rho}^{t-r} d \beta \int_{t-r}^{t+r} \frac{\alpha-\beta}{8 r}\left(\frac{\alpha+2 \rho}{4 \rho}\right)^{-(p-1)}\left\{\bar{u}\left(\frac{\alpha-\beta}{2}, \frac{\alpha+\beta}{2}\right)\right\}^{p} d \alpha
$$

for $0 \leq r \leq t-\rho$.

We divide the proof into two cases, $p \neq \frac{3}{2}$ and $p=\frac{3}{2}$.

(i) Estimation in the case of $p \neq \frac{3}{2}$.

Substituting (2.6) into (2.20), we get

$$
\begin{aligned}
L\left[|u|^{p}\right](x, t) & \leq\|u\|^{p} \int_{-\rho}^{t-r} d \beta \int_{t-r}^{t+r} \frac{\alpha-\beta}{8 r}\left(\frac{\alpha+2 \rho}{4 \rho}\right)^{-(p-1)}\left(\frac{\alpha+2 \rho}{\rho}\right)^{-p q}\left(\frac{\beta+2 \rho}{\rho}\right)^{-p \bar{q}} d \alpha \\
(2.21) & \leq 2^{2(p-1)} \rho^{2 p^{2}-p-1}\|u\|^{p} \times \frac{1}{r} \int_{t-r}^{t+r}(\alpha+2 \rho)^{-p(q+1)+2} d \alpha \times \int_{-\rho}^{t-r}(\beta+2 \rho)^{-p \bar{q}} d \beta .
\end{aligned}
$$


We evaluate the $\alpha$-integral in (2.21). For the case $t-r+2 \rho \geq \frac{1}{2}(t+r+2 \rho)$, we get

$$
\begin{aligned}
\frac{1}{r} \int_{t-r}^{t+r}(\alpha+2 \rho)^{-p(q+1)+2} d \alpha & \leq \frac{C}{r} \int_{t-r}^{t+r} d \alpha \times(t+r+2 \rho)^{-p(q+1)+2} \\
& \leq\left\{\begin{array}{ll}
C(t+r+2 \rho)^{-2 p^{2}+p+2} & \left(1<p<\frac{3}{2}\right) \\
C(t+r+2 \rho)^{-1}(t-r+2 \rho)^{-(2 p-3)} & \left(p>\frac{3}{2}\right)
\end{array} .\right.
\end{aligned}
$$

Next, we consider $t-r+2 \rho \leq \frac{1}{2}(t+r+2 \rho)$. In other words, for $t+2 \rho \leq 3 r$, we obtain

$$
\frac{1}{r} \int_{t-r}^{t+r}(\alpha+2 \rho)^{-p(q+1)+2} d \alpha \leq \frac{4}{t+r+2 \rho} \times\left\{\begin{array}{ll}
\frac{1}{\left(-2 p^{2}+p+3\right)}(t+r+2 \rho)^{-2 p^{2}+p+3} & \left(1<p<\frac{3}{2}\right) \\
\frac{1}{2 p-3}(t-r+2 \rho)^{-(2 p-3)} & \left(p>\frac{3}{2}\right)
\end{array} .\right.
$$

It follows from (2.22) and (2.23)

$$
\frac{1}{r} \int_{t-r}^{t+r}(\alpha+2 \rho)^{-p(q+1)+2} d \alpha \leq\left\{\begin{array}{ll}
C(t+r+2 \rho)^{-2 p^{2}+p+2} & \left(1<p<\frac{3}{2}\right) \\
C(t+r+2 \rho)^{-1}(t-r+2 \rho)^{-(2 p-3)} & \left(p>\frac{3}{2}\right)
\end{array} .\right.
$$

We evaluate the $\beta$-integral in (2.21). We get from (2.5)

$$
\int_{-\rho}^{t-r}(\beta+2 \rho)^{-p \bar{q}} d \beta \leq\left\{\begin{array}{ll}
t-r+\rho & \left(1<p<\frac{3}{2}\right) \\
\frac{2}{\gamma(p, 5)}(t-r+2 \rho)^{\gamma(p, 5) / 2} & \left(\frac{3}{2}<p<p_{S}(5)\right) \\
\log \frac{t-r+2 \rho}{\rho} & \left(p=p_{S}(5)\right) \\
C \rho^{\gamma(p, 5) / 2} & \left(p>p_{S}(5)\right)
\end{array} .\right.
$$

Hence, from (2.21), (2.24), (2.25) and (2.8), it follows that

$$
L\left[|u|^{p}\right](x, t) \leq C \rho^{2}\|u\|^{p}\left(\frac{t+r+2 \rho}{\rho}\right)^{-q}\left(\frac{t-r+2 \rho}{\rho}\right)^{-\bar{q}} D(T) \text { in } S_{2} .
$$

(ii) Estimation in the case of $p=\frac{3}{2}$.

Substituting (2.6) into (2.20), we get

$$
\begin{aligned}
L\left[|u|^{p}\right](x, t) & \leq\|u\|^{\frac{3}{2}} \int_{-\rho}^{t-r} d \beta \int_{t-r}^{t+r} \frac{\alpha-\beta}{8 r}\left(\frac{\alpha+2 \rho}{4 \rho}\right)^{-\frac{1}{2}}\left(\frac{\alpha+2 \rho}{\rho}\right)^{-\frac{3}{2}}\left\{\log \frac{2(\alpha+2 \rho)}{\beta+2 \rho}\right\}^{\frac{3}{2}} d \alpha \\
& \leq C \rho^{2}\|u\|^{\frac{3}{2}} \times \frac{1}{r} \int_{-\rho}^{t-r} d \beta \int_{t-r}^{t+r}(\alpha+2 \rho)^{-1}\left\{\log \frac{2(\alpha+2 \rho)}{\beta+2 \rho}\right\}^{\frac{3}{2}} d \alpha \\
(2.27) & \leq C \rho^{2}\|u\|^{\frac{3}{2}}\left\{J_{1}+J_{2}\right\},
\end{aligned}
$$

where

$$
\begin{aligned}
& J_{1}:=\left\{\log \frac{2(t+r+2 \rho)}{\rho}\right\}^{\frac{3}{2}} \times \frac{1}{r} \int_{-\rho}^{\rho} d \beta \int_{t-r}^{t+r}(\alpha+2 \rho)^{-1} d \alpha, \\
& J_{2}:=\frac{1}{r} \int_{\rho}^{t-r} d \beta \int_{t-r}^{t+r}(\alpha+2 \rho)^{-1}\left\{\log \frac{2(\alpha+2 \rho)}{\beta+2 \rho}\right\}^{\frac{3}{2}} d \alpha .
\end{aligned}
$$


By the same calculation as (2.22) and (2.23), we have

$$
\begin{aligned}
J_{1} & \leq C\left\{\log \frac{2(t+r+2 \rho)}{\rho}\right\}^{\frac{3}{2}}\left(\frac{t+r+2 \rho}{\rho}\right)^{-1} \log \frac{2(t+r+2 \rho)}{t-r+2 \rho} \\
& \leq C w(r, t)^{-1}\left\{\log \frac{2(2 T+3 \rho)}{\rho}\right\}^{\frac{3}{2}} .
\end{aligned}
$$

Next we evaluate $J_{2}$. Introducing new variables of integration $\sigma, \theta$ in $J_{2}$ by

$$
2(\alpha+2 \rho)=(t-r+2 \rho) \sigma, \quad \beta+2 \rho=(t-r+2 \rho) \theta \sigma
$$

and setting

$$
A=t+r+2 \rho, \quad B=t-r+2 \rho,
$$

we have

$$
\begin{aligned}
J_{2} & =\frac{B}{r} \int_{2}^{\frac{2 A}{B}} d \sigma \int_{\frac{3 \rho}{B \sigma}}^{\frac{1}{\sigma}}(-\log \theta)^{\frac{3}{2}} d \theta \\
& \leq \frac{2(A-B)}{r} \int_{0}^{1}(-\log \theta)^{\frac{3}{2}} d \theta \\
& \leq C .
\end{aligned}
$$

We obtain from (2.27), (2.28), (2.29) and (2.8)

$$
L\left[|u|^{p}\right](x, t) \leq C \rho^{2}\|u\|^{\frac{3}{2}} w(r, t)^{-1} D(T) \quad \text { in } S_{2} .
$$

Hence, from (2.26) and (2.30), we get (2.11) for $(r, t) \in S_{2}$. This completes the proof.

Proof of theorem 1.1. We define

$$
X=\left\{\begin{array}{l|l}
u(x, t) & \begin{array}{l}
D_{x}^{\alpha} u(x, t) \in C\left(\mathbb{R}^{3} \times[0, \infty)\right), \quad\left\|D_{x}^{\alpha} u\right\|<\infty \quad(|\alpha| \leq m(p)) \\
u(x, t)=0 \quad(|x| \geq t+\rho)
\end{array}
\end{array}\right\},
$$

where $D_{x}^{\alpha}=D_{1}^{\alpha_{1}} D_{2}^{\alpha_{2}} D_{3}^{\alpha_{3}}\left(\alpha=\left(\alpha_{1}, \alpha_{2}, \alpha_{3}\right)\right)$ and $D_{k}=\frac{\partial}{\partial x_{k}}(k=1,2,3)$. We can verify easily that $X$ is complete with respect to the norm

$$
\|u\|_{X}=\sum_{|\alpha| \leq m(p)}\left\|D_{x}^{\alpha} u\right\|
$$

Using the iteration method, we shall construct a solution of (1.10). We define the sequence of functions $\left\{u_{n}\right\}$ by

$$
u_{0}=u^{0}, \quad u_{n+1}=u^{0}+L\left[\left|u_{n}\right|^{p}\right] \quad \text { for } \quad n \geq 0 .
$$


We have from (2.1), (2.2) and (2.3)

$$
\left\|D^{\alpha} u^{0}\right\| \leq \epsilon \gamma 3^{2(p-1)} \rho^{-\nu} N_{\nu} \quad(|\alpha|=\nu \leq 3) .
$$

As in [13], p.258, we see from Lemma 2.2 and (2.31) that if $u^{0}$ satisfies

$$
C_{1} \rho^{2}\left\|u^{0}\right\|^{p-1} \leq \frac{1}{p 2^{p}},
$$

then $\left\{u_{n}\right\}$ is a Cauchy sequence in $X$. Since $X$ is complete, there exists a function $u \in X$ such that $D_{x}^{\alpha} u_{n}$ converges uniformly to $D_{x}^{\alpha} u, n \rightarrow \infty$. Clearly $u$ satisfies (1.10). In view of (1.10) and (1.11), we note that $\partial u / \partial t$ can be expressed in $D_{x}^{\alpha} u(|\alpha| \leq 1)$. Thus, from (2.31) and (2.32), Theorem 1.1 is proved by taking $\epsilon$ is small.

To prove Theorem 1.3, the following variant to the a priori estimate is required.

Lemma 2.3. Let $1<p \leq p_{S}(5)$ and $0 \leq \nu \leq p-1$. Assume that $u^{0}, u \in C\left(\mathbb{R}^{3} \times[0, T)\right)$ with (2.2) and supp $u \subset\left\{(x, t) \in \mathbb{R}^{3} \times[0, T)|| x \mid \leq t+\rho\right\}$. It holds

$$
\left\|L\left[\left|u^{0}\right|^{p-\nu}|u|^{\nu}\right]\right\| \leq C \rho^{2}\left\|u^{0}\right\|_{0}^{p-\nu}\|u\|^{\nu} \times\left\{\begin{array}{ll}
D(T)^{\frac{2 \nu(-2 p+3)}{\gamma(p, 5)}} & \left(1<p<\frac{3}{2}\right) \\
\left\{\log \frac{6(T+\rho)}{\rho}\right\}^{\nu} & \left(p=\frac{3}{2}\right) \\
1 & \left(\frac{3}{2}<p \leq p_{S}(5)\right)
\end{array},\right.
$$

where

$$
\left\|u^{0}\right\|_{0}:=\sup _{\substack{x \in \mathbb{R}^{3} \\ 0 \leq t<\infty}} \rho^{-1}(t+|x|+2 \rho)\left|u^{0}(x, t)\right| .
$$

Proof. From (2.34) and (2.2), we have

$$
0 \leq \overline{u^{0}}(\lambda, s) \leq\left\|u^{0}\right\|_{0}\left(\frac{s+\lambda+2 \rho}{\rho}\right)^{-1}
$$

and

$$
\overline{u^{0}}(\lambda, s)=0 \quad \text { for } \quad|\lambda-s| \geq \rho .
$$

To obtain (2.33), we show

$$
L\left[\left|u^{0}\right|^{p-\nu}|u|^{\nu}\right](x, t) \leq C \rho^{2}\left\|u^{0}\right\|_{0}^{p-\nu}\|u\|^{\nu} w(r, t)^{-1} \times\left\{\begin{array}{ll}
D(T)^{\frac{2 \nu(-2 p+3)}{\gamma(p, 5)}} & \left(1<p<\frac{3}{2}\right) \\
\left\{\log \frac{6(T+\rho)}{\rho}\right\}^{\nu} & \left(p=\frac{3}{2}\right) \\
1 & \left(\frac{3}{2}<p \leq p_{S}(5)\right)
\end{array} .\right.
$$


First, we show (2.37) for $(r, t) \in S_{1}$ defined by (2.12). We get from (2.35), (2.13) and (2.14)

$$
\overline{u^{0}}(\lambda, s)^{p-\nu} \bar{u}(\lambda, s)^{\nu} \leq\left\|u^{0}\right\|_{0}^{p-\nu}\|u\|^{\nu} \eta(t)^{\nu}\left(\frac{s+\rho}{\rho}\right)^{-p+\nu(1-q)} \quad \text { in } S_{1},
$$

where $\eta(t)$ is defined by (2.15).

By the same way as in (2.10), we obtain

$$
L\left[\left|u^{0}\right|^{p-\nu}|u|^{\nu}\right](x, t) \leq \iint_{\tilde{R}(r, t)} \frac{\lambda}{2 r}\left(\frac{s+\rho}{\rho}\right)^{-(p-1)} \overline{u_{0}}(\lambda, s)^{p-\nu} \bar{u}(\lambda, s)^{\nu} d \lambda d s .
$$

For $(r, t) \in S_{1}$, noticing $\tilde{R}(r, t) \subset S_{1}$ and substituting (2.38) into (2.39), we obtain from (2.16) and (2.13)

$$
\begin{aligned}
L\left[\left|u^{0}\right|^{p-\nu}|u|^{\nu}\right](x, t) & \leq\left\|u^{0}\right\|_{0}^{p-\nu}\|u\|^{\nu} \eta(t)^{\nu} \int_{0}^{t} d s\left(\frac{s+\rho}{\rho}\right)^{-2 p+1+\nu(1-q)} \int_{I} \frac{\lambda}{2 r} d \lambda \\
& \leq \frac{12 \rho^{2}\left\|u^{0}\right\|_{0}^{p-\nu}\|u\|^{\nu} \eta(t)^{\nu}}{t+\rho} \int_{0}^{t}\left(\frac{s+\rho}{\rho}\right)^{-2(p-1)+\nu(1-q)} d s \\
& \leq C \rho^{2}\left\|u^{0}\right\|_{0}^{p-\nu}\|u\|^{\nu} \eta(t)^{\nu+1}\left(\frac{t+\rho}{\rho}\right)^{-1+\max \{-2 p+3+\nu(1-q), 0\}} \\
& \leq C\left\|u^{0}\right\|_{0}^{p-\nu}\|u\|^{\nu} \rho^{2} w(r, t)^{-1} \times\left\{\begin{array}{ll}
\left(\frac{T+\rho}{\rho}\right)^{\nu(-2 p+3)} & \left(1<p<\frac{3}{2}\right) \\
\left\{\log \frac{6(T+\rho)}{\rho}\right\}^{\nu} & \left(p=\frac{3}{2}\right) \\
1 & \left(\frac{3}{2}<p \leq p_{S}(5)\right)
\end{array} .\right.
\end{aligned}
$$

Hence we obatin (2.37) for $(r, t) \in S_{1}$.

Next, we show (2.37) for $(r, t) \in S_{2}$ defined by (2.12).

By the same way as in (2.20), we get from (2.6), (2.35) and (2.36)

$$
\begin{aligned}
L\left[\left|u^{0}\right|^{p-\nu}|u|^{\nu}\right](x, t) & \leq\left\|u^{0}\right\|_{0}^{p-\nu}\|u\|^{\nu} \eta(t)^{\nu} \int_{-\rho}^{\rho} d \beta \int_{t-r}^{t+r} \frac{\alpha-\beta}{8 r}\left(\frac{\alpha+2 \rho}{4 \rho}\right)^{-(p-1)} \\
& \times\left(\frac{\alpha+2 \rho}{\rho}\right)^{-(p-\nu)-q \nu}\left(\frac{\beta+2 \rho}{\rho}\right)^{-\bar{q} \nu} d \alpha \\
& \leq 2^{2 p-5} \rho^{2 p-1-\nu(1-q-\bar{q})}\left\|u^{0}\right\|_{0}^{p-\nu}\|u\|^{\nu} \eta(t)^{\nu} \times \frac{1}{r} \int_{t-r}^{t+r}(\alpha+2 \rho)^{-2(p-1)+\nu(1-q)} d \alpha \\
& \times \int_{-\rho}^{\rho}(\beta+2 \rho)^{-\bar{q} \nu} d \beta \\
& \leq C \rho^{2 p-\nu(1-q)}\left\|u^{0}\right\|_{0}^{p-\nu}\|u\|^{\nu} \eta(t)^{\nu} \times \frac{1}{r} \int_{t-r}^{t+r}(\alpha+2 \rho)^{-2(p-1)+\nu(1-q)} d \alpha .
\end{aligned}
$$

By the same calculation as (2.22) and (2.23), we obtain

$$
\frac{1}{r} \int_{t-r}^{t+r}(\alpha+2 \rho)^{-2(p-1)+\nu(1-q)} d \alpha \leq\left\{\begin{array}{ll}
C(t+r+2 \rho)^{-2(p-1)+\nu(1-q)} & \left(p \neq \frac{3}{2}\right) \\
C(t+r+2 \rho)^{-1} \log \frac{2(t+r+2 \rho)}{t-r+2 \rho} & \left(p=\frac{3}{2}\right)
\end{array} .\right.
$$


We have from (2.41) and (2.42)

$$
L\left[\left|u^{0}\right|^{p-\nu}|u|^{\nu}\right](x, t) \leq C \rho^{2}\left\|u^{0}\right\|_{0}^{p-\nu}\|u\|^{\nu} w(r, t)^{-1} \times\left\{\begin{array}{ll}
\left(\frac{2 T+3 \rho}{\rho}\right)^{\nu(-2 p+3)} & \left(1<p<\frac{3}{2}\right) \\
\left\{\log \frac{6(T+\rho)}{\rho}\right\}^{\nu} & \left(p=\frac{3}{2}\right) \\
1 & \left(\frac{3}{2}<p \leq p_{S}(5)\right)
\end{array} .\right.
$$

Therefore, we get (2.37) for $(r, t) \in S_{2}$. This completes the proof.

From Lemma 2.3, we obtain the following estimate.

Lemma 2.4. Suppose that the assumptions in Lemma 2.3 are fulfilled. Then we have

$$
\left\|L\left[\left|u^{0}\right|^{p-\nu}|u|^{\nu}\right]\right\| \leq C_{2} \rho^{2}\left\|u^{0}\right\|_{0}^{p-\nu}\|u\|^{\nu} \times\left\{\begin{array}{ll}
1 & (\nu=0) \\
D(T)^{1 / p} & (\nu=1) \\
D(T)^{(p-1) /(p+1)} & (\nu=p-1)
\end{array} .\right.
$$

Proof. When $\frac{3}{2} \leq p \leq p_{S}(5)$, from Lemma 2.3 and (2.8), the estimate (2.44) is trivial. When $1<p<\frac{3}{2}$, we see that $\frac{2(-2 p+3)}{\gamma(p, 5)} \leq \frac{1}{p+1}$. Hence, from Lemma 2.3 and (2.8), we get (2.44). This completes the proof.

Proof of Theorem 1.3. We consider the following integral equation:

$$
U=L\left[\left|u^{0}+U\right|^{p}\right] \quad \text { in } \quad \mathbb{R}^{3} \times[0, T) .
$$

Suppose we obtain the solution of (2.45). Then, putting $u=U+u^{0}$, we get the solution of (1.10), and its lifespan is the same as that of $U$. Thus we have reduced the problem to the analysis of (2.45). Let $Y$ be the norm space defined by

$$
Y=\left\{\begin{array}{l|l}
U(x, t) & \begin{array}{l}
D_{x}^{\alpha} U(x, t) \in C\left(\mathbb{R}^{3} \times[0, T)\right), \quad\left\|D_{x}^{\alpha} U\right\|<\infty \quad(|\alpha| \leq 1) \\
U(x, t)=0 \quad(|x| \geq t+\rho)
\end{array}
\end{array}\right\}
$$

which is equipped with the norm

$$
\|U\|_{Y}=\sum_{|\alpha| \leq 1}\left\|D_{x}^{\alpha} U\right\|
$$

We shall construct a solution of the integral equation (2.45) in $Y$. We define the sequence of functions $\left\{U_{n}\right\}$ by

$$
U_{0}=0, \quad U_{n+1}=L\left[\left|u^{0}+U_{n}\right|^{p}\right] \quad(n=0,1,2, \cdots) .
$$

From Lemma 2.1, (2.13) and (2.34), we see that there exists a positive constant $C_{0}$ such that

$$
\left\|D_{x}^{\alpha} u^{0}\right\|_{0} \leq C_{0} \epsilon \quad(|\alpha| \leq 1)
$$


We put

$$
C_{3}:=\left(2^{2 p+2} p\right)^{\frac{p}{p-1}} \max \left\{C_{1} \rho^{2} M_{0}^{p-1},\left(C_{2} \rho^{2} C_{0}^{p-1}\right)^{p},\left(C_{2} \rho^{2} M_{0}^{p-2} C_{0}\right)^{\frac{p}{p-1}}\right\}
$$

and

$$
M_{0}:=2^{p} p \rho^{2} C_{0}^{p} \max \left\{C_{1}, C_{2}\right\}
$$

where $C_{1}$ and $C_{2}$ are constants given in Lemma 2.2 and Lemma 2.4. Analogously to the proof of Theorem 1 in [12, from Lemma 2.2 and Lemma 2.4, we see that $\left\{U_{n}\right\}$ is a Cauchy sequence in $Y$, if

$$
C_{3} \epsilon^{p(p-1)} D(T) \leq 1
$$

holds (see (4.3) in [12]). We can verify easily that $Y$ is complete. Hence, there exists a function $U$ such that $U_{n}$ converges to $U$ in $Y$. Therefore $U$ satisfies the integral equation (2.45).

Let us fix $\epsilon_{0}$ as

$$
C_{3} \epsilon_{0}^{p(p-1)} \leq \begin{cases}6^{-\gamma(p, 5) / 2} & \left(1<p<p_{S}(5)\right) \\ (\log 4)^{-1} & \left(p=p_{S}(5)\right)\end{cases}
$$

For $0<\epsilon \leq \epsilon_{0}$, if we assume that

$$
C_{3} \epsilon^{p(p-1)} \leq\left\{\begin{array}{l}
\left(\frac{4 T}{\rho}\right)^{-\gamma(p, 5) / 2} \quad\left(1<p<p_{S}(5)\right) \\
\left(\log \frac{2 T}{\rho}\right)^{-1} \quad\left(p=p_{S}(5)\right)
\end{array}\right.
$$

then (2.46) holds. Hence the lower bound estimate (1.14) follows immediately from (2.48). This completes the proof.

\section{Upper bound of the lifespan}

In this section, we prove Theorem 1.4. Our proof is based on the iteration argument which was introduced by [13. For the critical case, we apply the slicing method which was introduced by [6]. We show that the soluiton of (1.10) blows up in finite time.

Let $\widetilde{v}(r, t)$ be the spherical mean of $v \in C\left(\mathbb{R}^{3} \times[0, \infty)\right)$;

$$
\widetilde{v}(r, t)=\frac{1}{4 \pi} \int_{|\xi|=1} v(r \xi, t) d \omega_{\xi}
$$

We get the following representation formula (3.2) (for the proof, see [13]). 
Lemma 3.1. Let $L$ be the linear integral operator defined by (1.11). Assume that $v \in$ $C\left(\mathbb{R}^{3} \times[0, T)\right)$. Then, For $(r, t) \in[0, \infty) \times[0, T)$, it holds

$$
\widetilde{L[v]}(r, t)=\iint_{R(r, t)} \frac{\lambda}{2 r}(1+s)^{-(p-1)} \widetilde{v}(\lambda, s) d \lambda
$$

where

$$
R(r, t)=\{(\lambda, s) \mid t-r \leq s+\lambda \leq t+r, s-\lambda \leq t-r, s \geq 0\}
$$

Since $p>1,|u|^{p}$ is a convex function with $u$. By using the Jensen's inequality and Lemma 3.1, it follows from (1.10) that

$$
\widetilde{u}(r, t) \geq \widetilde{u^{0}}(r, t)+\iint_{R(r, t)} \frac{\lambda}{2 r}(1+s)^{-(p-1)}|\widetilde{u}(\lambda, s)|^{p} d \lambda .
$$

We define the following domains:

$$
\Sigma_{j}=\left\{(r, t) \mid l_{j} \rho \leq t-r \leq r\right\}, \quad \Sigma_{\infty}=\{(r, t) \mid 2 \rho \leq t-r \leq r\}
$$

where

$$
l_{j}=1+\frac{1}{2}+\cdots+\frac{1}{2^{j}} \quad(j=0,1,2, \cdots) .
$$

We derive a lower bound of the solution to (1.10), which is a first step of our iteration argument.

Lemma 3.2. We assume that $f \equiv 0$ and $g \geq 0(g \not \equiv 0)$. Let $u$ be a solution of (1.10). Then there exists a positive constant $M$ independent of $\epsilon$ such that

$$
\tilde{u}(r, t) \geq \frac{M \epsilon^{p}}{(t+r)(t-r)^{2 p-3}} \quad \text { in } \quad \Sigma_{0} .
$$

ProOF. From (1.12) and (3.1), the spherical averages $\widetilde{u^{0}}(r, t)$ satisfy $\left(\frac{\partial^{2}}{\partial t^{2}}-\frac{\partial^{2}}{\partial r^{2}}\right) r \widetilde{u^{0}}(r, t)=$ 0 . By using the d'Alembert's formula and the assumption $f \equiv 0$, we obtain

$$
\widetilde{u^{0}}(r, t)=\frac{H(t+r)-H(t-r)}{2 r}
$$

where

$$
H(s)=-\epsilon \int_{s}^{\infty} \sigma \widetilde{g}(\sigma) d \sigma .
$$

From (1.3), we have $H(s)=0$ for $s \geq \rho$. Since $g(x) \geq 0(\not \equiv 0)$, there exist constants $M_{0}>0, a, b(0<a<b<\rho)$ such that

$$
H(s) \leq-2 M_{0} \epsilon \quad(a \leq s \leq b) .
$$


From (3.7), for $t+r \geq \rho, a \leq t-r \leq b$, we get

$$
\begin{aligned}
\widetilde{u^{0}}(r, t) & =-\frac{H(t-r)}{2 r} \\
& \geq \frac{M_{0} \epsilon}{r} .
\end{aligned}
$$

We put

$$
S(r, t)=\{(\lambda, s) \mid t-r \leq \lambda, s+\lambda \leq 3(t-r), a \leq s-\lambda \leq b\} .
$$

For $(r, t) \in \Sigma_{0}$, we see $t+r \geq 3(t-r)$. Then, it follows that

$$
S(r, t) \subset\{(\lambda, s) \mid s+\lambda \geq \rho, a \leq s-\lambda \leq b\} \quad \text { in } \Sigma_{0} .
$$

Then, for $(r, t) \in \Sigma_{0}$, we have from (3.4), (3.8) and (3.9)

$$
\widetilde{u}(\lambda, s) \geq \frac{M_{0} \epsilon}{\lambda} \quad \text { in } S(r, t) .
$$

Noticing that $S(r, t) \subset R(r, t)$ for $(r, t) \in \Sigma_{0}$ and substituting (3.10) into (3.4), we get

$$
\tilde{u}(r, t) \geq \iint_{S(r, t)} \frac{\lambda}{2 r}(1+s)^{-(p-1)}\left|\frac{M_{0} \epsilon}{\lambda}\right|^{p} d \lambda d s \quad \text { in } \Sigma_{0} .
$$

Using the variables of integration $\alpha, \beta$ from (2.18) and since $t-r \geq \rho \geq 1$, we get

$$
\begin{aligned}
\tilde{u}(r, t) & \geq \frac{M_{0}^{p} \epsilon^{p}}{4 r} \int_{a}^{b} d \beta \int_{2(t-r)+\beta}^{3(t-r)}\left(\frac{\alpha-\beta}{2}\right)^{1-p}\left(\frac{2+\alpha+\beta}{2}\right)^{1-p} d \alpha \\
& \geq \frac{M_{0}^{p} \epsilon^{p}}{4 r} \int_{a}^{b} d \beta \int_{2(t-r)+\beta}^{3(t-r)}(3(t-r))^{1-p}(6(t-r))^{1-p} d \alpha \\
& =\frac{18^{-p} M_{0}^{p} \epsilon^{p}}{4 r(t-r)^{2(p-1)}} \int_{a}^{b}(t-r-\beta) d \beta \\
& \geq \frac{18^{-p}(b-a) M_{0}^{p} \epsilon^{p}(t-r-b)}{2(t+r)(t-r)^{2(p-1)}} \\
& \geq \frac{M \epsilon^{p}}{(t+r)(t-r)^{2 p-3}} \text { in } \Sigma_{0},
\end{aligned}
$$

where $M=2^{-1} 18^{-p}(b-a)(1-b / \rho) M_{0}^{p}$. This completes the proof.

Proof of Theorem 1.4. For $(r, t) \in \Sigma_{0}$, we introduce the sets

$$
Q_{j}(r, t)=\left\{(\lambda, s) \mid t-r \leq \lambda, s+\lambda \leq 3(t-r), l_{j} \rho \leq s-\lambda \leq t-r\right\} \quad(j=0,1,2, \cdots) .
$$

For $(r, t) \in \Sigma_{0}$, we see $t+r \geq 3(t-r)$. Then, we have

$$
\begin{aligned}
& Q_{j}(r, t) \subset R(r, t) \quad \text { in } \Sigma_{0}, \\
& Q_{j}(r, t) \subset \Sigma_{j} \text { in } \Sigma_{0},
\end{aligned}
$$


where $R(r, t)$ and $\Sigma_{j}$ are defined by (3.3) and (3.5).

Since $\Sigma_{j} \subset \Sigma_{0}$, we have from (3.4) and (3.11)

$$
\widetilde{u}(r, t) \geq \iint_{Q_{j}(r, t)} \frac{\lambda}{2 r}(1+s)^{-(p-1)}|\widetilde{u}(\lambda, s)|^{p} d \lambda \quad \text { in } \Sigma_{j} .
$$

We divide the proof into two cases, $1<p<p_{S}(5)$ and $p=p_{S}(5)$.

(i) Estimation in the case of $1<p<p_{S}(5)$.

We define the sequences $\left\{a_{j}\right\},\left\{b_{j}\right\}$ and $\left\{D_{j}\right\}$ by

$$
a_{0}=1, \quad b_{0}=2(p-1), \quad D_{0}=M \epsilon^{p}
$$

and

$$
a_{j+1}=p a_{j}+2, \quad b_{j+1}=p b_{j}+2(p-1), \quad D_{j+1}=\frac{18^{-p}}{2 a_{j+1}^{2}} D_{j}^{p} \quad(j=0,1,2, \cdots) .
$$

By using the induction argument, we derive

$$
\tilde{u}(r, t) \geq \frac{D_{j}(t-r-\rho)^{a_{j}}}{(t+r)(t-r)^{b_{j}}} \quad \text { in } \Sigma_{0} \quad(j=0,1,2, \cdots) .
$$

From (3.6), it holds (3.16) with $j=0$. We assume that (3.16) holds for one natural number $j$ and $(r, t) \in \Sigma_{0}$. Noticing that $Q_{0}(r, t) \subset \Sigma_{0}$ for $(r, t) \in \Sigma_{0}$ and putting (3.16) into (3.13), we get

$$
\tilde{u}(r, t) \geq \iint_{Q_{0}(r, t)} \frac{\lambda}{t+r}(1+s)^{-(p-1)}\left|\frac{D_{j}(s-\lambda-\rho)^{a_{j}}}{(s+\lambda)(s-\lambda)^{b_{j}}}\right|^{p} d \lambda d s \quad \text { in } \Sigma_{0} .
$$

Then using the variable of integration $\alpha, \beta$ from (2.18), we obtain

$$
\begin{aligned}
\widetilde{u}(r, t) & \geq \frac{1}{2(t+r)} \int_{\rho}^{t-r} d \beta \int_{2(t-r)+\beta}^{3(t-r)} \frac{\alpha-\beta}{2}\left(\frac{\alpha+\beta+2}{2}\right)^{1-p}\left|\frac{D_{j}(\beta-\rho)^{a_{j}}}{\alpha \beta^{b_{j}}}\right|^{p} d \alpha \\
& \geq \frac{D_{j}^{p}}{4(t+r)}\left((6(t-r))^{-(p-1)} \int_{\rho}^{t-r} d \beta \beta^{-p b_{j}}(\beta-\rho)^{p a_{j}} \int_{2(t-r)+\beta}^{3(t-r)} \frac{\alpha-\beta}{\alpha^{p}} d \alpha\right. \\
& \geq \frac{D_{j}^{p}}{4(t+r)}(6(t-r))^{-(p-1)}(t-r)^{-p b_{j}} \int_{\rho}^{t-r} d \beta(\beta-\rho)^{p a_{j}} \int_{2(t-r)+\beta}^{3(t-r)} \frac{2(t-r)}{3^{p}(t-r)^{p}} d \alpha \\
& =\frac{18^{-p} D_{j}^{p}}{2(t+r)(t-r)^{p b_{j}+2(p-1)}} \int_{\rho}^{t-r}(\beta-\rho)^{p a_{j}}(t-r-\beta) d \beta .
\end{aligned}
$$

Using integration by parts, it follows that

$$
\begin{aligned}
\int_{\rho}^{t-r}(\beta-\rho)^{p a_{j}}(t-r-\beta) d \beta & =\frac{1}{p a_{j}+1} \int_{\rho}^{t-r}(\beta-\rho)^{p a_{j}+1} d \beta \\
& \geq \frac{1}{a_{j+1}^{2}}(t-r-\rho)^{p a_{j}+2}
\end{aligned}
$$


Therefore, from (3.18) and (3.19), (3.16) holds for all natural number.

Solving (3.14) and (3.15) yields

$$
a_{j}=\frac{p^{j}(p+1)-2}{p-1}, \quad b_{j}=2\left(p^{j+1}-1\right) . \quad(j=0,1,2, \cdots) .
$$

Hence, we get from (3.15) and (3.20)

$$
D_{j+1} \geq \frac{F D_{j}^{p}}{p^{2(j+1)}}
$$

where $F=\frac{18^{-p}}{2}\left(\frac{p-1}{p+1}\right)^{2}$. Hence we have

$$
\log D_{j} \geq p^{j}\left[\log D_{0}+\sum_{k=1}^{j} \frac{p^{k-1} \log F-2 k \log p}{p^{j}}\right] .
$$

By using the d'Alembert's criterion, we see that the sum part in (3.21) converges as $j \rightarrow \infty$. Hence, from (3.14), there exists a constant $q$ such that it holds

$$
D_{j} \geq \exp \left\{p^{j} \log \left(M e^{q} \epsilon^{p}\right)\right\}
$$

Therefore, we have from (3.16), (3.20) and (3.22)

$$
\widetilde{u}(r, t) \geq \frac{\exp \left[p^{j} J(r, t)\right](t-r)^{2}}{(t+r)(t-r-\rho)^{\frac{2}{p-1}}} \text { in } \Sigma_{0} .
$$

Here,

$$
J(r, t)=\log \left\{\epsilon^{p} M e^{q} \frac{(t-r-\rho)^{\frac{p+1}{p-1}}}{(t-r)^{2 p}}\right\} .
$$

We take $\epsilon_{0}>0$ so small that

$$
B \epsilon_{0}^{-\frac{2 p(p-1)}{\gamma(p, 5)}} \geq 8 \rho
$$

where

$$
B=\left(2^{\frac{2\left(p^{2}-2 p-1\right)}{p-1}} M e^{q}\right)^{-\frac{2(p-1)}{\gamma(p, 5)}} .
$$

Next, for a fixed $\epsilon \in\left(0, \epsilon_{0}\right]$, we suppose that

$$
\tau>B \epsilon^{-\frac{2 p(p-1)}{\gamma(p, 5)}} \geq 8 \rho .
$$

Let $(r, t)=(\tau / 2, \tau)$. Then $(r, t) \in \Sigma_{0}$ and $t-r-2 \rho \geq(t-r) / 2$. Hence we get from (3.24) and (3.25)

$$
J(\tau / 2, \tau) \geq \log \left(\epsilon^{p}\left(B^{-1} \tau\right)^{\frac{\gamma(p, 5)}{2(p-1)}}\right)>0
$$


Therefore, from (3.23) and (3.26), we get $\tilde{u}(\tau / 2, \tau) \rightarrow \infty(j \rightarrow \infty)$. Hence, $T(\epsilon) \leq$ $B \epsilon^{-\frac{2 p(p-1)}{\gamma(p, 5)}}$ for $0<\epsilon \leq \epsilon_{0}$.

(ii) Estimation in the case of $p=p_{S}(5)$.

We define the sequences $\left\{d_{j}\right\}$ and $\left\{E_{j}\right\}$ by

$$
d_{0}=0, \quad E_{0}=M \epsilon^{p}
$$

and

$$
d_{j+1}=p d_{j}+1, \quad E_{j+1}=\frac{18^{-p} E_{j}^{p}}{2^{j+3} d_{j+1}} \quad(j=0,1,2, \cdots) .
$$

First, by using the induction argument, we will show

$$
\widetilde{u}(r, t) \geq \frac{E_{j}}{(t+r)(t-r)^{2 p-3}}\left(\log \frac{t-r}{l_{j} \rho}\right)^{d_{j}} \quad \text { in } \Sigma_{j} \quad(j=0,1,2, \cdots) .
$$

From (3.6), it holds (3.29) with $j=0$. We assume that (3.29) holds for one natural number $j$ and $(r, t) \in \Sigma_{j+1}$. From $p=p_{S}(5)$, we have $p(2 p-3)=1$. Noticing (3.12) and substituting (3.29) into (3.13), we obtain

$$
\begin{aligned}
\widetilde{u}(r, t) & \geq \frac{1}{2(t+r)} \int_{l_{j} \rho}^{t-r} d \beta \int_{2(t-r)+\beta}^{3(t-r)} \frac{\alpha-\beta}{2}\left(\frac{2+\alpha+\beta}{2}\right)^{1-p}\left|\frac{E_{j}}{\alpha \beta^{2 p-3}}\left(\log \frac{\beta}{l_{j} \rho}\right)^{d_{j}}\right|^{p} d \alpha \\
& \geq \frac{E_{j}^{p}}{2(t+r)} \int_{l_{j} \rho}^{t-r} d \beta \int_{2(t-r)+\beta}^{3(t-r)} \frac{6^{1-p}(t-r)^{1-p}}{3^{p}(t-r)^{p-1} \beta^{p(2 p-3)}}\left(\log \frac{\beta}{l_{j} \rho}\right)^{p d_{j}} d \alpha \\
& \geq \frac{18^{-p} E_{j}^{p}}{2(t+r)(t-r)^{2(p-1)}} \int_{l_{j} \rho}^{t-r} \frac{t-r-\beta}{\beta}\left(\log \frac{\beta}{l_{j} \rho}\right)^{p d_{j}} d \beta .
\end{aligned}
$$

Since $\rho \leq \frac{t-r}{l_{j+1}}$, we get from (3.30) and (3.28)

$$
\begin{aligned}
& \widetilde{u}(r, t) \geq \frac{18^{-p} E_{j}^{p}}{2(t+r)(t-r)^{2(p-1)}} \int_{l_{j} \rho}^{t-r}(t-r-\beta)\left[\frac{1}{p d_{j}+1}\left(\log \frac{\beta}{l_{j} \rho}\right)^{p d_{j}+1}\right]^{\prime} d \beta \\
& =\frac{18^{-p} E_{j}^{p}}{2(t+r)(t-r)^{2(p-1)}} \times \frac{1}{p d_{j}+1} \int_{l_{j} \rho}^{t-r}\left(\log \frac{\beta}{l_{j} \rho}\right)^{p d_{j}+1} d \beta \\
& \geq \frac{18^{-p} E_{j}^{p}}{2 d_{j+1}(t+r)(t-r)^{2(p-1)}} \int_{\frac{l_{j}(t-r)}{l_{j+1}}}^{t-r}\left(\log \frac{\beta}{l_{j} \rho}\right)^{d_{j+1}} d \beta \\
& \geq \frac{18^{-p}\left(1-\frac{l_{j}}{l_{j+1}}\right) E_{j}^{p}}{2 d_{j+1}(t+r)(t-r)^{2 p-3}}\left(\log \frac{t-r}{l_{j+1} \rho}\right)^{d_{j+1}} \text {. }
\end{aligned}
$$

From $1-\frac{l_{j}}{l_{j+1}}=\frac{1}{2^{j+1} l_{j+1}} \geq \frac{1}{2^{j+2}}$, we get

$$
\begin{aligned}
\widetilde{u}(r, t) & \geq \frac{18^{-p} E_{j}^{p}}{2^{j+3} d_{j+1}(t+r)(t-r)^{2 p-3}}\left(\log \frac{t-r}{l_{j+1} \rho}\right)^{d_{j+1}} \\
& =\frac{E_{j+1}}{(t+r)(t-r)^{2 p-3}}\left(\log \frac{t-r}{l_{j+1} \rho}\right)^{d_{j+1}} \text { in } \Sigma_{j+1} .
\end{aligned}
$$


Therefore, (3.29) holds for all natural number.

Solving (3.27) and (3.28) yields

$$
d_{j}=\frac{p^{j}-1}{p-1} \quad(j=0,1,2, \cdots) .
$$

Hence, we get

$$
E_{j+1} \geq \frac{G E_{j}^{p}}{(2 p)^{j}}
$$

where $G=\frac{18^{-p}(p-1)}{2^{3} p}$. Therefore, it follows that

$$
\log E_{j} \geq p^{j}\left[\log E_{0}+\sum_{k=1}^{j} \frac{p^{k-1} \log G-(k-1) \log (2 p)}{p^{j}}\right] .
$$

The sum part in (3.32) converges as $j \rightarrow \infty$ by the d'Alembert's criterion. Hence, there exists a constant $q$ such that it holds from (3.27)

$$
E_{j} \geq \exp \left\{p^{j} \log \left(M e^{q} \epsilon^{p}\right)\right\} .
$$

Since $\Sigma_{\infty} \subset \Sigma_{j}$ and $l_{j} \leq 2$, we obtain from (3.29) and (3.31)

$$
\begin{aligned}
\widetilde{u}(r, t) & \geq \frac{\exp \left\{p^{j} \log \left(M e^{q} \epsilon^{p}\right)\right\}}{(t+r)(t-r)^{2 p-3}}\left(\log \frac{t-r}{2 \rho}\right)^{\frac{p^{j}-1}{p-1}} \\
& \geq \frac{\exp \left\{p^{j} J(r, t)\right\}}{(t+r)(t-r)^{2 p-3}}\left(\log \frac{t-r}{2 \rho}\right)^{-\frac{1}{p-1}} \quad \text { in } \Sigma_{\infty},
\end{aligned}
$$

where

$$
J(r, t)=\log \left(\epsilon^{p}\left(B^{-1} \log \frac{t-r}{2 \rho}\right)^{\frac{1}{p-1}}\right), \quad B=\left(M e^{q}\right)^{-(p-1)} .
$$

We take $\epsilon_{0}>0$ so small that

$$
B \epsilon_{0}^{-p(p-1)} \geq \log (4 \rho)
$$

For a fixed $\epsilon \in\left(0, \epsilon_{0}\right]$, we suppose that $\tau$ satisfies

$$
\tau>\exp \left(2 B \epsilon^{-p(p-1)}\right)(>4 \rho) .
$$

From (3.36) and (3.35), it follows that

$$
\tau>4 \rho \exp \left(B \epsilon^{-p(p-1)}\right) .
$$

We get (3.34) and (3.37)

$$
J(\tau / 2, \tau)=\log \left(\epsilon^{p}\left(B^{-1} \log \frac{\tau}{4 \rho}\right)^{\frac{1}{p-1}}\right)>0 .
$$

Since $(\tau / 2, \tau) \in \Sigma_{\infty}$, from (3.33) and (3.38), we get $u(\tau / 2, \tau) \rightarrow \infty \quad(j \rightarrow \infty)$. Hence, $T(\epsilon) \leq \exp \left(2 B \epsilon^{-p(p-1)}\right)$ for $0<\epsilon \leq \epsilon_{0}$. This completes the proof. 
Acknowledement. The authors would like to express his sincere gratitude to Professor Hiroyuki Takamura for his valuable advices.

\section{References}

[1] M.D'Abbicco, The threshold of effective damping for semilinear wave equations, Math. Methods Appl. Sci. 38 (2015), no. 6, 1032-1045.

[2] M.D'Abbicco and S.Lucente, A modified test function method for damped wave equations, Adv. Nonlinear Stud. 13 (2013), no. 4, 867-892.

[3] M.D'Abbicco and S.Lucente, NLWE with a special scale invariant damping in odd space dimension, Dyn. Syst. Differential Equations Appl. AIMS Proc. (2015), 312319.

[4] M.D'Abbicco, S.Lucente and M.Reissig, Semi-linear wave equations with effective damping, Chin. Ann. Math. Ser. B 34 (2013), no. 3, 345-380.

[5] M.D'Abbicco, S.Lucente and M.Reissig, A shift in the Strauss exponent for semilinear wave equations with a not effective damping, J. Differential Equations 259 (2015), no. 10, 5040-5073.

[6] R.Agemi, Y.Kurokawa and H.Takamura, Critical curve for $p$-q systems of nonlinear wave equations in three space dimensions, J. Differential Equations 167 (2000), no. $1,87-133$.

[7] P.Almenar, L.Jódar and J.A.Martin, Mixed problems for the time-dependent telegraph equation: continuous numerical solutions with a priori error bounds., Math. Comput. Modelling 25 (1997), no. 11, 31-44.

[8] K.Fujiwara, M.Ikeda and Y.Wakasugi, Estimates of lifespan and blow-up rates for the wave equation with a time-dependent damping and a power-type nonlinearity, Funkcial. Ekvac. (in press) arXiv:1609.01035.

[9] M.Ikeda and T.Inui, The sharp estimate of the lifespan for the semilinear wave equation with time-dependent damping, arXiv:1707.03950.

[10] M.Ikeda and T.Ogawa, Lifespan of solutions to the damped wave equation with a critical nonlinearity, J. Differential Equations 261 (2016), no. 3, 1880-1903. 
[11] M.Ikeda, M.Sobajima, Life-span of solutions to semilinear wave equation with timedependent critical damping for specially localized initial data, Mathematische Annalen (in press) arXiv:1709.004401.

[12] T.Imai, M.Kato, H.Takamura, K.Wakasa, The sharp lower bound of the lifespan of solutions to semilinear wave equations with low powers in two space dimensions, in: Proceeding of the international conference Asymptotic Analysis for Nonlinear Dispersive and Wave Equations of a volume in Advanced Study of Pure Mathematics, (in press) arXiv:1610.05913.

[13] F.John, Blow-up of solutions of nonlinear wave equations in three space dimensions, Manuscripta Math. 28 (1979), 235-268.

[14] N.-A.Lai, H.Takamura, Blow-up for semilinear damped wave equations with subcritical exponent in the scattering case, Nonlinear Anal. 168 (2018), 222-237.

[15] N.-A.Lai, H.Takamura, K.Wakasa, Blow-up for semilinear wave equations with the scale invariant damping and super-Fujita exponent, J. Differential Equations 263 (2017), no. 9, 5377-5394.

[16] K.Wakasa, The lifespan of solutions to semilinear damped wave equations in one space dimension, Commun. Pure Appl. Anal. 15 (2016), no. 4, 1265-1283.

[17] Y.Wakasugi, Critical exponent for the semilinear wave equation with scale invariant damping, Fourier Analysis, Trends Math., Birkhuser/Springer, Cham (2014), 375390.

[18] Y.Wakasugi, On the Diffusive Structure for the Damped Wave Equation with Variable Coefficients (Doctoral thesis), Osaka University, 2014.

\section{KATO}

Muroran Institute of TeChnology

27-1 Mizumoto-Cho, Muroran 050-8585, JAPAN

E-mail:mkato@mmm.muroran-it.ac.jp

\section{SAKURABA}

SApporo Hokuto High School

1-10 Kita 15, Higashi 2, Higashi-Ku, SAPPORO 065-0015, JAPAN

E-mail: miku.sakuraba@gmail.com 